\title{
Centrifugal deformations of the gravitational kink
}

\author{
Paolo Maraner ${ }^{\mathrm{a}}$, Jiannis K. Pachos ${ }^{\mathrm{b}}$ \\ ${ }^{a}$ School of Economics and Management, Free University of Bozen-Bolzano, \\ via Sernesi 1, 39100 Bolzano, Italy \\ ${ }^{\mathrm{b}}$ School of Physics and Astronomy, University of Leeds, Leeds LS2 9JT, UK
}

\begin{abstract}
The Kaluza-Klein reduction of $4 \mathrm{~d}$ conformally flat spacetimes is reconsidered. The corresponding $3 \mathrm{~d}$ equations are shown to be equivalent to $2 \mathrm{~d}$ gravitational kink equations augmented by a centrifugal term. For space-like gauge fields and nontrivial values of the centrifugal term the gravitational kink solutions describe a spacetime that is divided in two disconnected regions.
\end{abstract}

Key words: Gravitational kinks, conformal flatness, Kaluza-Klein reduction.

\section{Introduction}

Reducing the gravitational Chern-Simons term from three to two dimensions produces an interesting as well as surprising outcome [1]. On the one hand the resulting equations of motion require the vanishing of the $3 \mathrm{~d}$ Cotton tensor and, hence, impose conformal flatness of the original $3 \mathrm{~d}$ spacetime. On the other hand, they take the form of general covariant kink equations that, besides symmetry breaking solutions, support an associated kink. Let us parameterize the $3 \mathrm{~d}$ line element as $d s_{(3)}^{2}=\mathrm{g}_{i j} d x^{i} d x^{j}+\left(\mathrm{A}_{i} d x^{i}+d x^{2}\right)^{2}$, where Latin indices range over 0,1 and all quantities are independent of the third coordinate $x^{2}$. Then, the equations obtained by Guralnik, Iorio, Jackiw and Pi $((4.47,48,49)$ in Ref. [1]) read

$$
\begin{aligned}
& \mathrm{R}=k+3 f^{2}, \\
& \mathrm{D}^{2} f+k f-f^{3}=0, \\
& \mathrm{D}_{i} \mathrm{D}_{j} f-\frac{1}{2} \mathrm{~g}_{i j} \mathrm{D}^{2} f=0 .
\end{aligned}
$$


Here $\mathrm{g}_{i j}$ is the $2 \mathrm{~d}$ spacetime metric, $\mathrm{D}_{i}$ is the associated covariant derivative, $\mathrm{D}^{2}=\mathrm{D}_{i} \mathrm{D}^{i}, \mathrm{R}$ is the relative scalar curvature and $k$ is an arbitrary constant. The Kaluza-Klein field strength $\mathrm{F}_{i j}=\partial_{i} \mathrm{~A}_{j}-\partial_{j} \mathrm{~A}_{i}$ has been expressed in terms of its dual scalar 1

$$
\mathrm{F}_{i j} \equiv \sqrt{-\mathrm{g}} \varepsilon_{i j} f
$$

Equation (1b) is the covariant generalization of the flat space kink equation with biquadratic potential

$$
V(f)=\frac{1}{4}\left(k-f^{2}\right)^{2}
$$

supporting, for positive values of $k$, the well known kink profile. Equations (1a) and (1c) appear instead, as subsidiary constraints necessary to uniquely determine the geometry $\mathrm{g}_{i j}$ together with the field $f$. As shown in Ref. [1], general covariant kinks bear a close relation to flat space kinks governed by identical potentials. Indeed, equations (1) support the solution

$$
\begin{aligned}
& \mathrm{g}_{i j} d x^{i} d x^{j}=-\frac{1}{4} k^{2} \operatorname{sech}^{4}\left(\frac{\sqrt{k}}{2} x^{1}\right)\left(d x^{0}\right)^{2}+\left(d x^{1}\right)^{2} \\
& \mathrm{~A}_{i} d x^{i}= \pm \frac{1}{2} k \operatorname{sech}^{2}\left(\frac{\sqrt{k}}{2} x^{1}\right) d x^{0}
\end{aligned}
$$

with the corresponding kink profile

$$
f= \pm \sqrt{k} \tanh \left(\frac{\sqrt{k}}{2} x^{1}\right)
$$

It is rather natural to wonder wether this situation generalizes to higher dimensions. Indeed, the Kaluza-Klein reduction of conformally flat spaces was recently considered by Grumiller and Jackiw for arbitrary dimensions [2]. The resulting, rather daunting, equations dramatically simplify for the reduction from $4 \mathrm{~d}$ to $3 \mathrm{~d}$. Grumiller and Jackiw restricted to that case and constructed special solutions based on a further Ansatz on the 3d metric. Here, we reconsider these equations in their generality. For space-like gauge fields, we show that the $3 \mathrm{~d}$ general covariant equations describing the Kaluza-Klein reduction of $4 \mathrm{~d}$ conformally flat spaces correspond to the $2 \mathrm{~d}$ general covariant kink equations (11) supplemented by a centrifugal term. Hence, besides the $3 \mathrm{~d}$ extension of the gravitational kink (4), 4d conformally flat Kaluza-Klein spaces also support their 'centrifugal deformations'.

Our discussion proceeds as follows. In §2 we briefly review 3d Grumiller-Jackiw equations and supplement them with some extra considerations. In \$3 we

1 Some signs differ from Ref. [1], and in what follow from Ref. [2], due to different conventions. Here $\mathrm{R}_{i j k}{ }^{l}=\partial_{i} \Gamma_{j k}^{l}-\ldots, \mathrm{R}_{i j}=\mathrm{R}_{k i j}{ }^{k}, \mathrm{R}=\mathrm{R}_{i}{ }^{i}$ and analogously in other dimensions. The single time-like direction carries negative signature, while space-like directions carry positive signature. 
take advantage of general covariance to adapt coordinates and reduce the equations to $2 \mathrm{~d}$ centrifugal kink equations. The centrifugal deformations of the gravitational kink are eventually constructed in \$4.

\section{Grumiller-Jackiw equations in 3d}

Let us parameterize the $4 d$ line element as $d s_{(4)}^{2}=g_{\mu \nu} d x^{\mu} d x^{\nu}+\left(A_{\mu} d x^{\mu}+d x^{3}\right)^{2}$, where Greek indices range over $0,1,2$ and all quantities are independent of the last coordinate $x^{3}$. Then, the 3d Grumiller-Jackiw equation $22((22 \mathrm{a}, \mathrm{b})$ in Ref. [2]) read

$$
\begin{aligned}
& R_{\mu \nu}-\frac{1}{3} R g_{\mu \nu}=f_{\mu} f_{\nu}-\frac{1}{3} f_{\kappa} f^{\kappa} g_{\mu \nu} \\
& D_{\mu} f_{\nu}+D_{\nu} f_{\mu}=0
\end{aligned}
$$

Here, $g_{\mu \nu}$ is the $3 \mathrm{~d}$ spacetime metric, $D_{\mu}$ is the associated covariant derivative and $R_{\mu \nu}$ and $R$ are the corresponding Ricci and scalar curvatures. The KaluzaKlein field strength $F_{\mu \nu}=\partial_{\mu} A_{\nu}-\partial_{\nu} A_{\mu}$ has been reexpressed in terms of its dual vector

$$
F_{\mu \nu} \equiv \sqrt{-g} \varepsilon_{\mu \nu \kappa} f^{\kappa}
$$

Grumiller and Jackiw also derived two important consequences of equations $(5 \mathrm{~b})$ and (5b). First, the scalar curvature $R$ can be expressed in terms of $f_{\mu} f^{\mu}$ and an arbitrary constant $k$ as ((23) in Ref. [2])

$$
R=3 k-5 f_{\mu} f^{\mu} .
$$

Second, the vector $F^{\mu}=\frac{1}{\sqrt{-g}} \varepsilon^{\mu \nu \kappa} D_{\nu} f_{\kappa}$, when not identically vanishing, is a second Killing vector of the geometry ((26c) in Ref. [2])

$$
D_{\mu} F_{\nu}+D_{\nu} F_{\mu}=0
$$

Before proceeding it is useful to rewrite equation (8) in a slightly different form and to supplement equations (5a) and (5b) with a further integrability condition.

After rising both indices of (8) we reexpress $F^{\mu}$ in terms of $f^{\mu}$ obtaining

$$
\varepsilon^{\lambda \rho \sigma} D^{\kappa} D_{\rho} f_{\sigma}+\varepsilon^{\kappa \rho \sigma} D^{\lambda} D_{\rho} f_{\sigma}=0 .
$$

$\overline{2}$ In 3d, Grumiller-Jackiw equations are the Minkowskian analogue of the Euclidean Einstein-Weyl equations, widely studied and completely solved in mathematics (see [3, 4] and references therein). 
Contracting with $\varepsilon_{\lambda \mu \nu}$, reexpressing $\varepsilon$-symbols products in terms of Kronecker deltas and taking into account (5b) we immediately have

$$
D_{\kappa} D_{\mu} f_{\nu}-\frac{1}{2} g_{\kappa \mu} D^{2} f_{\nu}+\frac{1}{2} g_{\kappa \nu} D^{2} f_{\mu}=0
$$

closely resembling the traceless equation (1c).

To derive the integrability condition consider the covariant derivative of (5b)

$$
D_{\kappa} D_{\mu} f_{\nu}+D_{\kappa} D_{\nu} f_{\mu}=0
$$

Antisymmetrizing in $\kappa$ and $\mu$, contracting $\mu$ with $\nu$ and taking into account the vanishing of the covariant divergence of the gauge field, $D_{\mu} f^{\mu}=0$, we have

$$
\left[D_{\kappa}, D_{\mu}\right] f^{\mu}-D_{\mu} D^{\mu} f_{\kappa}=0
$$

Expressing the commutator of covariant derivatives in terms of the Ricci tensor and inserting (5a) and (7), we eventually obtain the integrability condition

$$
D^{2} f^{\mu}+k f^{\mu}-f_{\nu} f^{\nu} f^{\mu}=0
$$

closely resembling the gravitational kink equation (1b). The similarity between the sets of equations (7), (9), (10) and (1a), (1c), (1b) is made even stronger by properly adapted coordinates.

\section{Darboux coordinates}

By an appropriate coordinate transformation we now set $f^{0}=f^{1}=0$, i.e. $F_{12}=F_{20}=0$. The existence of such a coordinate frame is guarantied by a classical result in symplectic geometry, Darboux theorem [5]. In 3d it ensures the possibility of finding, in a finite neighborhood of every point, local coordinates in such a way that a given closed two-form (a $U(1)$ gauge field) can be rewritten, e.g., as

$$
F_{\mu \nu}=\left(\begin{array}{cc}
\mathrm{F}_{i j} & 0 \\
0 & 0
\end{array}\right)
$$

with $i, j=0,1$ and $\mathrm{F}_{i j}$ a closed $2 \mathrm{~d}$ two-form. Darboux coordinates are determined up to the reparametrization $x^{i} \rightarrow x^{\prime i}\left(x^{0}, x^{1}\right), x^{2} \rightarrow x^{\prime 2}\left(x^{0}, x^{1}, x^{2}\right)$, so that we still have a certain freedom in choosing them. In the adapted frames the metric takes an arbitrary form. Without loss of generality it can be parameterized as

$$
g_{\mu \nu} d x^{\mu} d x^{\nu}=\mathrm{g}_{i j} d x^{i} d x^{j}+\mathrm{h}\left(\mathrm{a}_{i} d x^{i}+d x^{2}\right)^{2}
$$


where $\mathrm{g}_{i j}, \mathrm{a}_{i}$ and $\mathrm{h}$, are arbitrary functions of all coordinates 3 Under the residual covariance group, $\mathrm{g}_{i j}$ transforms like a $2 \mathrm{~d}$ metric tensor, $\mathrm{a}_{i}$ identifies with a $2 \mathrm{~d}$ gauge potential taking values in the $1 \mathrm{~d}$ diffeomorphism algebra, while $\mathrm{h}$ transforms like a scalar. For $f^{\mu}$ space-like, $\mathrm{g}_{i j}$ is Minkowskian, h definite positive and time can be identified with $x^{0}$. For $f^{\mu}$ time-like, $\mathrm{g}_{i j}$ is Euclidean, $\mathrm{h}$ definite negative and time has to be identified with $x^{2}$. In any case, we proceed as in $\S 1$ and re-express the effectively $2 \mathrm{~d}$ gauge field $\mathrm{F}_{i j}=\partial_{i} \mathrm{~A}_{j}-\partial_{j} \mathrm{~A}_{i}$ in terms of its dual scalar 4

$$
\mathrm{F}_{i j} \equiv \sqrt{|\mathrm{g}|} \varepsilon_{i j} f
$$

Consequently, in adapted coordinates, the $3 \mathrm{~d}$ gauge field $f^{\mu}$ rewrites as

$$
f^{\mu}=\left(0,0,|\mathrm{~h}|^{-\frac{1}{2}} f\right) .
$$

We also introduce the gauge curvature $\mathrm{f}_{i j}$ associated to the, in general, nonabelian vector potential $\mathrm{a}_{i}$

$$
\mathrm{f}_{i j}=\partial_{i} \mathrm{a}_{j}-\partial_{j} \mathrm{a}_{i}-\mathrm{a}_{i} \partial_{2} \mathrm{a}_{j}+\mathrm{a}_{j} \partial_{2} \mathrm{a}_{i}
$$

and the relative dual scalar

$$
\mathrm{f}_{i j} \equiv \sqrt{|\mathrm{g}|} \varepsilon_{i j} \mathrm{f}
$$

Eventually, we take advantage of residual covariance by rescaling $x^{2}$ in such a way that

$$
\mathrm{h}=\mathrm{h}\left(x^{0}, x^{1}\right)
$$

Correspondingly, covariance is reduced to arbitrary redefinitions of $x^{i}, x^{i} \rightarrow$ $x^{i}\left(x^{0}, x^{1}\right)$, and linear redefinitions of $x^{2}, x^{2} \rightarrow \xi\left(x^{0}, x^{1}\right) x^{2}$.

We now proceed by rewriting equations (5), (9) and (10) in adapted Darboux coordinates. The most convenient starting point is equation (5b). Its $i j, i 2$ and 22 components can be rearranged into the lower dimensional covariant equations

$$
\begin{aligned}
& \partial_{2} \mathrm{~g}_{i j}=0, \\
& \partial_{2} \mathrm{a}_{i}=\frac{1}{2} \partial_{i} \ln \left(|\mathrm{h}| f^{-2}\right), \\
& \partial_{2} f=0 .
\end{aligned}
$$

3 We remark that, in spite of the similarity, this is not a further Kaluza-Klein Ansatz as the metric entries depend on the 'extra' coordinate $x^{2}$.

4 With the only exceptions of coordinates and of the gauge field $f, 2 \mathrm{~d}$ quantities are expressed in Roman characters. 
The first and third clearly require

$$
\mathrm{g}_{i j}=\mathrm{g}_{i j}\left(x^{0}, x^{1}\right), \quad f=f\left(x^{0}, x^{1}\right),
$$

while the integration of the second one produces

$$
\mathrm{a}_{i}=\frac{1}{2} \partial_{i} \ln \left(|\mathrm{h}| f^{-2}\right) x^{2}+\overline{\mathrm{a}}_{i}\left(x^{0}, x^{1}\right)
$$

with $\overline{\mathrm{a}}_{i}\left(x^{0}, x^{1}\right)$ an arbitrary $2 \mathrm{~d}$ one-form. The gauge potential, $\mathrm{a}_{i}$, is at most linear in the 'extra' coordinate $x^{2}$. As a consequence, we can take further advantage of residual covariance to eliminate the $x^{2}$ dependence. In fact, the coordinate transformation $x^{2} \rightarrow \frac{1}{2} \ln \left(|\mathrm{h}| f^{-2}\right) x^{2}$ causes the off-diagonal blocks of the metric to transform as $\mathrm{a}_{i} \rightarrow \mathrm{a}_{i}-\frac{1}{2} \partial_{i} \ln \left(|\mathrm{h}| f^{-2}\right) x^{2}$, making the transformed gauge potential to be independent of $x^{2}$,

$$
\mathrm{a}_{i}=\mathrm{a}_{i}\left(x^{0}, x^{1}\right)
$$

In the new adapted coordinates we have $\partial_{2} \mathrm{a}_{i}=0$ so equation (18b) implies the proportionality between $\mathrm{h}$ and $f^{2}$. The relative scale factor can be fixed to any non-zero value by a constant rescaling of $x^{2}$. It is positive/negative when $f^{\mu}$ is space/time-like. For definiteness, we fix the scale factor to be equal to \pm 2 , i.e.

$$
\mathrm{h}= \pm 2 f^{2}
$$

Here and in the following, upper/lower signs refer to space/time-like $f^{\mu}$. The 'extra' coordinate $x^{2}$ is now completely fixed and residual covariance is reduced to $2 \mathrm{~d}$ general covariance, $x^{i} \rightarrow x^{i}\left(x^{0}, x^{1}\right)$.

Next, we consider the integrability condition (10). Taking (19), (20) and (21) into account, its $i$ and 2 components become

$$
\begin{aligned}
& \partial_{i}\left(f^{3} \mathrm{f}\right)=0, \\
& \mathrm{D}^{2} f+k f \mp f^{3}+f^{3} \mathrm{f}^{2}=0,
\end{aligned}
$$

with $\mathrm{D}^{2}=\mathrm{D}_{i} \mathrm{D}^{i}$ and $\mathrm{D}_{i}$ the covariant derivative associated to the $2 \mathrm{~d}$ metric $\mathrm{g}_{i j}$. The first equation requires the product $f^{3} \mathrm{f}$ to be constant, allowing to reexpress $\mathrm{f}$ in terms of $f$. We set

$$
f^{3} \mathrm{f}=l
$$

with $l$ an arbitrary constant. Consequently, (22b) reduces to

$$
\mathrm{D}^{2} f+k f \mp f^{3}+\frac{l^{2}}{f^{3}}=0 .
$$

For space-like $f^{\mu}$, (24a) is the gravitational kink equation (1b) augmented by a centrifugal term of 'angular momentum' $l$. Eventually, we consider equations 
(5a) and (9) in view of (19), (20), (21) and (23). Equation (5a) reduces to

$$
\mathrm{R}=k \mp 3 f^{2}-\frac{3 l^{2}}{f^{4}}
$$

for $\mathrm{R}$ the $2 \mathrm{~d}$ scalar curvature associated to $\mathrm{g}_{i j}$. For space-like $f^{\mu}$, (24b) corresponds to the curvature constraint (1b) up to the 'centrifugal' term $-3 l^{2} / f^{4}$. Equation (9) reduces instead to

$$
\mathrm{D}_{i} \mathrm{D}_{j} f-\frac{1}{2} \mathrm{~g}_{i j} \mathrm{D}^{2} f=0
$$

corresponding to the traceless equation (1c) without any modification.

Equations (24) are completely equivalent to equations (15).

\section{Centrifugal kinks}

We now specialize to space-like gauge fields. For $l=0$ equations (24) exactly correspond to the gravitational kink of equations (1). Therefore, they support a 3d gravitational kink, with $\mathrm{g}_{i j}$ given by (4a), $\mathrm{A}_{i}$ by (4b), $\mathrm{a}_{i}=0$ and $\mathrm{h}$ equal to twice the square of (4c). The resulting $3 \mathrm{~d}$ spacetime is a warped product of the $2 \mathrm{~d}$ kink spacetime with the real line, where the warp factor is twice the square of the kink profile. For $l \neq 0$, the biquadratic potential (3) is supplemented by the centrifugal term $l^{2} /\left(2 f^{2}\right)$. Correspondingly, the scalar curvature $\mathrm{R}$ is augmented by $-3 l^{2} / f^{4}=-\left(l^{2} /\left(2 f^{2}\right)\right)^{\prime \prime}$ (see Appendix B of Ref. [1]). By properly choosing the integration constant the new potential can be written as

$$
V(f)=\frac{\left(\kappa-\lambda-f^{2}\right)^{2}\left(f^{2}+\lambda\right)}{4 f^{2}}
$$

with the constants $\kappa$ and $\lambda$ related to $k$ and $l$ by $k=\kappa-3 \lambda / 2$ and $2 l^{2}=$ $(\kappa-\lambda)^{2} \lambda$. The integration of the corresponding flat space equation (see Appendices A and B of Ref. [1) is immediate and leads to the following deformation of the general covariant kink

$$
\begin{aligned}
& \mathrm{g}_{i j} d x^{i} d x^{j}=-\frac{\kappa^{3} \operatorname{sech}^{4}\left(\frac{\sqrt{\kappa}}{2} x^{1}\right) \tanh ^{2}\left(\frac{\sqrt{\kappa}}{2} x^{1}\right)}{4 \kappa \tanh ^{2}\left(\frac{\sqrt{\kappa}}{2} x^{1}\right)-4 \lambda}\left(d x^{0}\right)^{2}+\left(d x^{1}\right)^{2} \\
& \mathrm{a}_{i} d x^{i}= \pm \frac{\kappa \sqrt{2 \lambda}}{4(\kappa-\lambda) \cosh ^{2}\left(\frac{\sqrt{\kappa}}{2} x^{1}\right)-4 \kappa} d x^{0} \\
& \mathrm{~h}=2 \kappa \tanh ^{2}\left(\frac{\sqrt{\kappa}}{2} x^{1}\right)-2 \lambda \\
& \mathrm{A}_{i} d x^{i}= \pm \frac{\kappa}{2} \operatorname{sech}^{2}\left(\frac{\sqrt{\kappa}}{2} x^{1}\right) d x^{0}
\end{aligned}
$$


with the corresponding centrifugal distortion of the kink profile

$$
f= \pm \sqrt{\kappa \tanh ^{2}\left(\frac{\sqrt{\kappa}}{2} x^{1}\right)-\lambda}
$$

For $l=0$ the constants $\kappa$ and $\lambda$ respectively reduce to $k$ and 0 , so that (26) correctly reproduces the $3 \mathrm{~d}$ gravitational kink. For $l \neq 0$ the $2 \mathrm{~d}$ metric $\mathrm{g}_{i j}$, and the corresponding scalar curvature $\mathrm{R}$, is singular at $x^{1}= \pm \frac{2}{\sqrt{\kappa}} \operatorname{arctanh} \sqrt{\frac{\lambda}{\kappa}}$, while the gauge field $f$ is only defined for $\left|x^{1}\right|>\frac{2}{\sqrt{\kappa}} \operatorname{arctanh} \sqrt{\frac{\lambda}{\kappa}}$. The effect of the centrifugal interaction is that of breaking the kink in two parts, pushing them apart, and thus dividing spacetime in two disconnected regions.

\section{Acknowledgments}

We would like to thank Daniel Grumiller and Roman Jackiw for inspiring conversations. This work was supported by the Royal Society.

\section{References}

[1] G. Guralnik, A. Iorio, R. Jackiw, S.-Y. Pi, Dimensionally reduced gravitational Chern-Simons term and its kink, Ann. Phys. 308 (2003) 222-236.

[2] D. Grumiller, R. Jackiw, Kaluza-Klein reduction of conformally flat spaces, Int. J. Mod. Phys. D15 (2006) 2075-2094.

[3] R. Jackiw, Dimensional reduction of conformal tensors and Einstein-Weyl spaces, SIGMA 3 (2007) 91-97.

[4] D. Grumiller, R. Jackiw, Einstein-Weyl from Kaluza-Klein, Phys. Lett. A372 (2008) 2547-2551.

[5] See e.g. D. Mc Duff, D. Salamon, Introduction to Symplectic Topology, Oxford University Press (1998). 